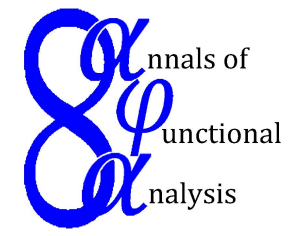

Ann. Funct. Anal. 6 (2015), no. 2, 234-241

http://doi.org/10.15352/afa/06-2-20

ISSN: 2008-8752 (electronic)

http://projecteuclid.org/afa

\title{
FURTHER REFINEMENTS OF ZHAN'S INEQUALITY FOR UNITARILY INVARIANT NORMS
}

\author{
HONGLIANG ZUO*1, YUKI SEO² AND MASATOSHI FUJII ${ }^{2}$ \\ Communicated by M. S. Moslehian
}

\begin{abstract}
In this paper, we show a further improvement of the integral Heinz mean inequality and prove

$\frac{1}{2}\left\|A^{2} X+2 A X B+X B^{2}\right\| \leq \frac{2}{t+2}\left\|A^{2} X+t A X B+X B^{2}\right\| \|$ for all $t \in(-2,2]$.
\end{abstract}

Then we show some refinements of unitarily invariant norm inequalities, in particular we proved that: If $A, B, X \in M_{n}$ with $A$ and $B$ positive definite, and $f, g$ are two continuous functions on $(0, \infty)$ such that $h(x)=\frac{f(x)}{g(x)}$ is Kwong, then

$$
\left\|A^{\frac{1}{2}}(f(A) X g(B)+g(A) X f(B)) B^{\frac{1}{2}}\right\| \leq \frac{k}{2}\left\|A^{2} X+2 A X B+X B^{2}\right\|
$$

holds for any unitarily invariant norm, where $k=\max \left\{\frac{f(\lambda) g(\lambda)}{\lambda} \mid \lambda \in \sigma(A) \cup \sigma(B)\right\}$.

\section{INTRODUCTION}

A capital letter means an $n \times n$ matrix in the matrix algebra $M_{n},\|\cdot\| \cdot \|$ denotes a unitarily invariant norm on $M_{n}$, that is, $\|U A V\|=\|A\|$ for all matrices $A, U, V$ with $U, V$ unitary, and $\sigma(A)$ is the set of all eigenvalues of $A \in M_{n}$. The Schur product of $A=\left(a_{i j}\right) \in M_{n}$ and $B=\left(b_{i j}\right) \in M_{n}$ is denoted by $A \circ B$, i.e., $A \circ B=\left(a_{i j} b_{i j}\right)$.

The following double inequality due to Bhatia and Davis (see [2]) asserts that

$$
2\left\|A^{\frac{1}{2}} X B^{\frac{1}{2}}\right\| \leq\|\| A^{r} X B^{1-r}+A^{1-r} X B^{r}\|\leq\| A X+X B \|
$$

for $A, B, X \in M_{n}$ with $A, B$ positive semidefinite and $0 \leq r \leq 1$. Afterwards, Wang et al. [10] proved that

$$
2\|A X B\| \leq\left\|A^{r} X B^{2-r}+A^{2-r} X B^{r}\right\| \| \text { for } 1 \leq 2 r \leq 3 .
$$

Date: Received: Aug. 31, 2014; Accepted: Sep. 16, 2014.

* Corresponding author.

2010 Mathematics Subject Classification. Primary 15A60; Secondary 47A63, 47A30.

Key words and phrases. Heinz inequality, Zhan's inequality, Hermite-Hadamard inequality, unitarily invariant norm. 
Recently Kaur et al. [4] showed the following refinement of the Hermite-Hadamard inequality which improved the left-hand side of (1.1).

Let $A, B, X \in M_{n}$ with $A, B$ positive semidefinite. Then, for any real numbers $\alpha, \beta$, we have

$$
\begin{aligned}
\| A^{\frac{\alpha+\beta}{2}} & X B^{1-\frac{\alpha+\beta}{2}}+A^{1-\frac{\alpha+\beta}{2}} X B^{\frac{\alpha+\beta}{2}} \| \\
& \leq \frac{1}{|\alpha-\beta|}\left\|\int_{\alpha}^{\beta}\left(A^{v} X B^{1-v}+A^{1-v} X B^{v}\right) d v\right\| \| \\
& \leq \frac{1}{2}\left\|\mid A^{\alpha} X B^{1-\alpha}+A^{1-\alpha} X B^{\alpha}+A^{\beta} X B^{1-\beta}+A^{1-\beta} X B^{\beta}\right\| .
\end{aligned}
$$

The right-hand side of (1.1), called the Heinz inequality, was generalized by Zhan [11] in the following sense.

Theorem ZH. Let $A, B, X \in M_{n}$ with $A, B$ positive semidefinite. Then

$$
\left\|A^{r} X B^{2-r}+A^{2-r} X B^{r}\right\| \leq \frac{2}{t+2}\left\|A^{2} X+t A X B+X B^{2}\right\|
$$

for any real numbers $r, t$ satisfying $1 \leq 2 r \leq 3$ and $-2<t \leq 2$.

Singh and Vasudeva [9] showed that if $A, B, X \in M_{n}$ with $A, B$ positive definite, and $f$ is any matrix monotone function on $(0, \infty)$, then for $-2<t \leq 2$

$$
\left\|A^{\frac{1}{2}} f(A) X f(B)^{-1} B^{\frac{3}{2}}+A^{\frac{3}{2}} f(A)^{-1} X f(B) B^{\frac{1}{2}}\right\| \leq \frac{2}{t+2}\left\|A^{2} X+t A X B+X B^{2}\right\|
$$

A continuous real-valued function $f$ defined on an interval $(a, b)$ with $a \geq 0$ is called a Kwong function [1] if the matrix

$$
K_{f}=\left(\frac{f\left(\lambda_{i}\right)+f\left(\lambda_{j}\right)}{\lambda_{i}+\lambda_{j}}\right)_{i, j=1,2, \cdots, n}
$$

is positive semidefinite for any distinct real numbers $\lambda_{1}, \cdots, \lambda_{n}$ in $(a, b)$. It is easy to see that if $f$ is a non-zero Kwong function then $f$ is positive and $\frac{1}{f}$ is Kwong. Kwong [6] showed that the set of all Kwong functions on $(0, \infty)$ is a closed cone and includes all non-negative operator monotone functions on $(0, \infty)$. Also Audenaert [1] gave a characterization of Kwong functions by showing that, for fixed $0 \leq a<b$, a function $f$ on an interval $(a, b)$ is Kwong if and only if the function $g(x)=\sqrt{x} f(\sqrt{x})$ is operator monotone on $\left(a^{2}, b^{2}\right)$. Afterwards, Najafi [8] obtained a more generalized norm inequality of the Heinz inequality.

$$
\|f(A) X g(B)+g(A) X f(B)\| \leq\|A X+X B\|
$$

for any continous functions $f(x), g(x)$ with $\frac{f(x)}{g(x)}$ Kwong and $f(x) g(x) \leq x$.

For a comprehensive inspection of the results concerning the above norm inequalities, the reader is referred to $[3,5,7,10]$.

In this paper we show some improvements of the above inequalities (1.1)-(1.5). Especially, we prove that if $A, B, X \in M_{n}$ such that $A$ and $B$ are positive semidefinite, then

$$
\begin{aligned}
\left\|A^{r} X B^{2-r}+A^{2-r} X B^{r}\right\| & \leq \frac{1}{2}\left\|A^{2} X+2 A X B+X B^{2}\right\| \\
& \leq \frac{2}{t+2}\left\|A^{2} X+t A X B+X B^{2}\right\|
\end{aligned}
$$


holds for $1 \leq 2 r \leq 3$ and $-2<t \leq 2$.

\section{Refined ZhAN'S INEQUALITY}

In this section, we show a refined version of Zhan's inequality. First of all, we study the function at the right-hand side of inequality (1.4).

Theorem 2.1. Let $A, B, X \in M_{n}$ with $A, B$ positive semidefinite. Suppose that

$$
\Psi(t)=\frac{2}{t+2}\left\|A^{2} X+t A X B+X B^{2}\right\|, \quad t \in(-2,2] .
$$

Then $\Psi(t)$ is monotone increasing on $(-2,2]$. In particular,

$$
\begin{aligned}
\left\|A^{r} X B^{2-r}+A^{2-r} X B^{r}\right\| & \leq \frac{1}{2}\left\|A^{2} X+2 A X B+X B^{2}\right\| \\
& \leq \frac{2}{t+2}\left\|A^{2} X+t A X B+X B^{2}\right\|
\end{aligned}
$$

holds for $1 \leq 2 r \leq 3$ and $t \in(-2,2]$.

Proof. It's suffice to prove the monotonicity of $\Psi(t)$. For any $-2<s<t \leq 2$, there exists $\alpha \in(0,1)$ such that $\frac{2}{t+2}=\frac{2 \alpha}{s+2}$. Then we have

$$
\begin{aligned}
\Psi(t) & =\frac{2}{t+2}\left\|A^{2} X+t A X B+X B^{2}\right\| \\
& =\left\|\frac{2}{t+2}\left(A^{2} X+X B^{2}\right)+\left(1-\frac{2}{t+2}\right) 2 A X B\right\| \\
& =\left\|\frac{2 \alpha}{s+2}\left(A^{2} X+X B^{2}\right)+\left(1-\frac{2 \alpha}{s+2}\right) 2 A X B\right\| \| \\
& =\left\|\frac{2 \alpha}{s+2}\left(A^{2} X+s A X B+X B^{2}\right)+\left(1-\frac{2 \alpha}{s+2}-\frac{\alpha s}{s+2}\right) 2 A X B\right\| \| \\
& =\left\|\frac{2 \alpha}{s+2}\left(A^{2} X+s A X B+X B^{2}\right)+(1-\alpha) 2 A X B\right\| \| \\
& \left.\leq \frac{2 \alpha}{s+2} \| A^{2} X+s A X B+X B^{2}\right)\|+(1-\alpha) 2\| A X B \| \\
& \left.\leq \frac{2}{s+2} \| A^{2} X+s A X B+X B^{2}\right) \|=\Psi(s),
\end{aligned}
$$

where the last inequality follows from (1.2) and (1.4). Therefore, $\Psi(t)$ is monotone increasing on $(-2,2]$.

Besides, Zhan's inequality and the monotonicity of $\Psi(t)$ yield inequality (2.1).

Next we show a generalized Zhan's inequality. For this, we need the following lemma.

Lemma 2.2. Let $\sigma_{1}, \sigma_{2}, \cdots, \sigma_{n}$ be any positive real numbers. If $f$ and $g$ are two continuous functions on $(0, \infty)$ such that $h(x)=\frac{f(x)}{g(x)}$ is Kwong, then the $n \times n$ matrix

$$
W=\left(\frac{f\left(\sigma_{i}\right) g^{-1}\left(\sigma_{i}\right)+f\left(\sigma_{j}\right) g^{-1}\left(\sigma_{j}\right)}{\sigma_{i}^{2}+2 \sigma_{i} \sigma_{j}+\sigma_{j}^{2}}\right)_{i, j=1,2, \cdots, n}
$$

is positive semidefinite. 
Proof. Let $W=\left(w_{i j}\right)$. Applying the Schur product theorem to the Cauchy matrix $\left(\frac{1}{\sigma_{i}+\sigma_{j}}\right)$, and the matrix induced from the Kwong function $h$, we get the positivity of the matrix $W$ with entries

$$
w_{i j}=\frac{1}{\sigma_{i}+\sigma_{j}} \frac{f\left(\sigma_{i}\right) g^{-1}\left(\sigma_{i}\right)+f\left(\sigma_{j}\right) g^{-1}\left(\sigma_{j}\right)}{\sigma_{i}+\sigma_{j}} .
$$

Lemma 2.3. [5, p. 343] If $X=\left(x_{i j}\right)$ is positive semidefinite, then for any matrix $Y$,

$$
\|X \circ Y\| \leq \max _{1 \leq i \leq n} x_{i i}\|Y\| .
$$

Theorem 2.4. Suppose that $A, B, X \in M_{n}$ such that $A, B$ are positive definite, and $f$, $g$ are two continuous functions on $(0, \infty)$ such that $h(x)=\frac{f(x)}{g(x)}$ is Kwong. Then

$$
\left\|A^{\frac{1}{2}}(f(A) X g(B)+g(A) X f(B)) B^{\frac{1}{2}}\right\| \leq \frac{k}{2}\left\|A^{2} X+2 A X B+X B^{2}\right\|
$$

holds for $k=\max \left\{\frac{f(\lambda) g(\lambda)}{\lambda} \mid \lambda \in \sigma(A) \cup \sigma(B)\right\}$.

Proof. We first prove the special case $A=B$, i.e.,

$$
\left\|A^{\frac{1}{2}}[f(A) X g(A)+g(A) X f(A)] A^{\frac{1}{2}}\right\| \leq k\|\| \frac{1}{2}\left(A^{2} X+2 A X A+X A^{2}\right) \| .
$$

Without loss of generality, assume that $A$ is positive definite. Suppose that $A=$ $U \Sigma U^{*}$ is the spectral decomposition with $U$ unitary and $\Sigma=\operatorname{diag}\left(\sigma_{1}, \sigma_{2}, \cdots, \sigma_{n}\right)$ with $\sigma_{1}, \sigma_{2}, \cdots, \sigma_{n}>0$. The required inequality is equivalent to

$$
\left\|\Sigma^{\frac{1}{2}}\left[f(\Sigma) U^{*} X U g(\Sigma)+g(\Sigma) U^{*} X U f(\Sigma)\right] \Sigma^{\frac{1}{2}}\right\| \leq k\left\|\frac{1}{2}\left(\Sigma^{2} U^{*} X U+2 \Sigma U^{*} X U \Sigma+U^{*} X U \Sigma^{2}\right)\right\|,
$$

which may be rewritten as

$$
\left\|\left[\sigma_{i}^{\frac{1}{2}}\left(f\left(\sigma_{i}\right) g\left(\sigma_{j}\right)+g\left(\sigma_{i}\right) f\left(\sigma_{j}\right)\right) \sigma_{j}^{\frac{1}{2}} y_{i, j}\right]\right\|\|k\|\left[\frac{1}{2}\left(\sigma_{i}^{2}+2 \sigma_{i} \sigma_{j}+\sigma_{j}^{2}\right) y_{i, j}\right]\|\|
$$

for all $\left[y_{i, j}\right] \in M_{n}$. This is the same as

$$
\|W \circ Y\| \leq k\|Y\| \quad \text { for all } \quad Y \in M_{n},
$$

where

$$
W=2 \operatorname{diag}\left(\sigma_{\mathrm{i}}^{\frac{1}{2}} \mathrm{~g}\left(\sigma_{\mathrm{i}}\right)\right)\left(\frac{f\left(\sigma_{i}\right) g^{-1}\left(\sigma_{i}\right)+f\left(\sigma_{j}\right) g^{-1}\left(\sigma_{j}\right)}{\sigma_{i}^{2}+2 \sigma_{i} \sigma_{j}+\sigma_{j}^{2}}\right) \operatorname{diag}\left(\sigma_{\mathrm{j}}^{\frac{1}{2}} \mathrm{~g}\left(\sigma_{\mathrm{j}}\right)\right) \in M_{n} .
$$

In fact, we can conclude that $W$ is positive semidifinite by Lemma 2.2 Then it follows from Lemma 2.3 and $k=\max \left\{\frac{f(\lambda) g(\lambda)}{\lambda} \mid \lambda \in \sigma(A) \cup \sigma(B)\right\}$ that

$$
\|W \circ Y\| \leq \max _{1 \leq i \leq n} w_{i i}\|Y\|=k\|Y\|
$$

for all $Y \in M_{n}$. Hence (2.2) holds too.

For the general case, it suffices to replace $A$ and $X$ by

$$
\left[\begin{array}{cc}
A & 0 \\
0 & B
\end{array}\right] \text { and }\left[\begin{array}{cc}
0 & X \\
0 & 0
\end{array}\right] \text {, respectively. }
$$


Remark 2.5. Theorem 2.4 can be seen as a refinement of (1.5) if we let $f$ be a operator monotone function on $(0, \infty)$ and $g(x)=x f^{-1}(x)$. Then $x^{-1} f^{2}(x)$ is Kwong [6] and $k=\max \left\{\frac{f(\lambda) g(\lambda)}{\lambda} \mid \lambda \in \sigma(A) \cup \sigma(B)\right\}=1$. Hence, for $-2<t \leq 2$, we have

$$
\begin{aligned}
\left\|A^{\frac{1}{2}} f(A) X f(B)^{-1} B^{\frac{3}{2}}+A^{\frac{3}{2}} f(A)^{-1} X f(B) B^{\frac{1}{2}}\right\| & \leq \frac{1}{2}\left\|A^{2} X+2 A X B+X B^{2}\right\| \\
& \leq \frac{2}{t+2}\left\|A^{2} X+t A X B+X B^{2}\right\|
\end{aligned}
$$

holds for $A, B, X \in M_{n}$ with $A, B$ positive definite.

Using the method in the proof of Theorem 2.4 again we get the following theorem.

Theorem 2.6. Suppose that $A, B, X \in M_{n}$ such that $A, B$ are positive definite. If $f$ and $g$ are two continuous functions on $(0, \infty)$ such that $h(x)=\frac{f(x)}{g(x)}$ is Kwong, then

$$
\|f(A) X g(B)+g(A) X f(B)\| \leq \frac{k^{\prime}}{2}\left\|A^{2} X+2 A X B+X B^{2}\right\|
$$

holds for $k^{\prime}=\max \left\{\frac{f(\lambda) g(\lambda)}{\lambda^{2}} \mid \lambda \in \sigma(A) \cup \sigma(B)\right\}$.

Example 2.7. Take $f(x)=\log (1+x)$ and $g(x)=x$ defined on $(0, \infty)$. Then $f(x) g(x)^{-1}$ is Kwong but not operator monotone; cf. [8]. Theorem 2.4 leads to the following inequality:

$$
\left\|A^{\frac{1}{2}}(\log (I+A) X B+A X \log (I+B)) B^{\frac{1}{2}}\right\| \leq \frac{\log \left(1+\lambda_{0}\right)}{2}\left\|A^{2} X+2 A X B+X B^{2}\right\|
$$

for $A, B, X \in M_{n}$ with $A, B$ positive semidefinite, where $\lambda_{0}=\max \{\lambda \mid \lambda \in \sigma(A) \cup \sigma(B)\}$.

Example 2.8. Let $s, r \in \mathbb{R}$. Due to $F(x)=x^{s-r}$ defined on $(0, \infty)$ is Kwong if and only if $-1 \leq s-r \leq 1$, it follows from Theorem 2.4 that if $A, B, X \in M_{n}$ with $A, B$ positive semidefinite and $|s-r| \leq 1$, then

$$
\left\|A^{\frac{1}{2}}\left(A^{s} X B^{r}+A^{r} X B^{s}\right) B^{\frac{1}{2}}\right\| \leq \frac{k}{2}\left\|A^{2} X+2 A X B+X B^{2}\right\|
$$

for $k=\max \left\{\lambda^{s+r-1} \mid \lambda \in \sigma(A) \cup \sigma(B)\right\}$. In particular, if $1 \leq 2 r \leq 3$, put $r^{\prime}=r-\frac{1}{2}$, $s^{\prime}=\frac{3}{2}-r$ in the above inequality, then Zhan's inequality (1.4) follows.

\section{Refined integral Heinz mean inequality}

Now we prove a stronger matrix version of the integral Heinz mean by using the same integration technique as that in [4].

Theorem 3.1. Let $A, B, X \in M_{n}$ with $A$ and $B$ positive semidefinite. Then, for any positive real number $\alpha, \beta$, it holds that

$$
\begin{aligned}
& \frac{1}{|\alpha-\beta|}\left\|\int_{\alpha}^{\beta}\left(A^{v} X B^{1-v}+A^{1-v} X B^{v}\right) d v\right\| \| \\
& \quad \leq \frac{1}{4}\left\|2 A^{\frac{\alpha+\beta}{2}} X B^{1-\frac{\alpha+\beta}{2}}+2 A^{1-\frac{\alpha+\beta}{2}} X B^{\frac{\alpha+\beta}{2}}+A^{\alpha} X B^{1-\alpha}+A^{1-\alpha} X B^{\alpha}+A^{\beta} X B^{1-\beta}+A^{1-\beta} X B^{\beta}\right\| .
\end{aligned}
$$

Proof. It's enough to prove the special case $A=B$. Without loss of generality, let $A$ be positive definite and $\alpha<\beta$. Since the norm considered here is unitarily invariant, we can assume that $A$ is diagonal, i.e., $A=\operatorname{diag}\left(\sigma_{1}, \sigma_{2}, \cdots, \sigma_{n}\right), \sigma_{1} \geq \sigma_{2} \geq \cdots \geq \sigma_{n}>0$.

Note that

$$
\begin{aligned}
& \int_{\alpha}^{\beta}\left(A^{v} X A^{1-v}+A^{1-v} X A^{v}\right) d v \\
= & Z \circ\left(2 A^{\frac{\alpha+\beta}{2}} X A^{1-\frac{\alpha+\beta}{2}}+2 A^{1-\frac{\alpha+\beta}{2}} X A^{\frac{\alpha+\beta}{2}}+A^{\alpha} X A^{1-\alpha}+A^{1-\alpha} X A^{\alpha}+A^{\beta} X A^{1-\beta}+A^{1-\beta} X A^{\beta}\right),
\end{aligned}
$$


whence $Z=\left(z_{i, j}\right)$,

$$
\begin{aligned}
z_{i, j} & =\frac{\int_{\alpha}^{\beta}\left(\exp \left(\log \left(\sigma_{i}\right) v+\log \left(\sigma_{j}\right)(1-v)\right)+\exp \left(\log \left(\sigma_{i}\right)(1-v)+\log \left(\sigma_{j}\right) v\right)\right) d v}{2 \sigma_{i}^{\frac{\alpha+\beta}{2}} \sigma_{j}^{1-\frac{\alpha+\beta}{2}}+2 \sigma_{i}^{1-\frac{\alpha+\beta}{2}} \sigma_{j}^{\frac{\alpha+\beta}{2}}+\sigma_{i}^{\alpha} \sigma_{j}^{1-\alpha}+\sigma_{i}^{1-\alpha} \sigma_{j}^{\alpha}+\sigma_{i}^{\beta} \sigma_{j}^{1-\beta}+\sigma_{i}^{1-\beta} \sigma_{j}^{\beta}} \\
& =\frac{1}{\log \sigma_{i}-\log \sigma_{j}} \frac{\sigma_{i}^{\beta} \sigma_{j}^{1-\beta}-\sigma_{i}^{1-\beta} \sigma_{j}^{\beta}-\sigma_{i}^{\alpha} \sigma_{j}^{1-\alpha}+\sigma_{i}^{1-\alpha} \sigma_{j}^{\alpha}}{2 \sigma_{i}^{\frac{\alpha+\beta}{2}} \sigma_{j}^{1-\frac{\alpha+\beta}{2}}+2 \sigma_{i}^{1-\frac{\alpha+\beta}{2}} \sigma_{j}^{\frac{\alpha+\beta}{2}}+\sigma_{i}^{\alpha} \sigma_{j}^{1-\alpha}+\sigma_{i}^{1-\alpha} \sigma_{j}^{\alpha}+\sigma_{i}^{\beta} \sigma_{j}^{1-\beta}+\sigma_{i}^{1-\beta} \sigma_{j}^{\beta}} \\
& =\frac{1}{\log \sigma_{i}-\log \sigma_{j}} \frac{\left(\sigma_{i}^{\beta-\alpha}-\sigma_{j}^{\beta-\alpha}\right)\left(\sigma_{i}^{\alpha} \sigma_{j}^{1-\beta}+\sigma_{i}^{1-\beta} \sigma_{j}^{\alpha}\right)}{2 \sigma_{i}^{\frac{\beta-\alpha}{2}}\left(\sigma_{i}^{\alpha} \sigma_{j}^{1-\beta}+\sigma_{i}^{1-\beta} \sigma_{j}^{\alpha}\right) \sigma_{j}^{\frac{\beta-\alpha}{2}}+\left(\sigma_{i}^{\beta-\alpha}+\sigma_{j}^{\beta-\alpha}\right)\left(\sigma_{i}^{\alpha} \sigma_{j}^{1-\beta}+\sigma_{i}^{1-\beta} \sigma_{j}^{\alpha}\right)} \\
& =\frac{1}{\log \sigma_{i}-\log \sigma_{j}} \frac{\sigma_{i}^{\beta-\alpha}-\sigma_{j}^{\beta-\alpha}}{\left(\sigma_{i}^{\frac{\beta-\alpha}{2}}+\sigma_{j}^{\frac{\beta-\alpha}{2}}\right)^{2}}=\frac{1}{\log \sigma_{i}-\log \sigma_{j}} \frac{\sigma_{i}^{\frac{\beta-\alpha}{2}}-\sigma_{j}^{\frac{\beta-\alpha}{2}}}{\sigma_{i}^{\frac{\beta-\alpha}{2}}+\sigma_{j}^{\frac{\beta-\alpha}{2}}}, \text { for } i \neq j .
\end{aligned}
$$

and $z_{i, i}=\frac{\beta-\alpha}{4}$. On taking $\sigma_{i}^{\frac{\beta-\alpha}{2}}=\exp \left(t_{i}\right)$ we conclude that $Z$ is positive semidefinite if and only if so is the matrix with entries

$$
\frac{4}{\beta-\alpha} z_{i, j}^{\prime}= \begin{cases}\frac{\tanh \left(\left(t_{i}-t_{j}\right) / 2\right)}{\left(t_{i}-t_{j}\right) / 2}, & \text { if } i \neq j \\ 1, & \text { if } i=j\end{cases}
$$

The above matrix is positive semidefinite since the function $\frac{\tanh x}{x}$ is positive definite [2, p. 223].

In addition, since all diagonal entries of the matrix $Z$ are $\frac{\beta-\alpha}{4}$, we have $\|Z \circ Y\| \leq$ $\max _{1 \leq i \leq n} z_{i i}\|Y\|=\frac{\beta-\alpha}{4}\|Y\|$ for all $Y \in M_{n}$. Therefore, this proves the required inequality in Theorem 3.1 for the case of $A=B$.

Put $\alpha=r, \beta=2-r$ for any $r \in\left[\frac{1}{2}, \frac{3}{2}\right]$ and replace $A, B$ by $A^{2}, B^{2}$, respectively, in Theorem 3.1, then we achieve a refinement of inequality (1.2).

Corollary 3.2. Let $A, B, X \in M_{n}$ with $A$ and $B$ positive semidefinite and $r \in\left[\frac{1}{2}, \frac{3}{2}\right]$. Then

$$
\begin{aligned}
2\|A X B\| & \leq \frac{1}{|2-2 r|}\left\|\int_{r}^{2-r}\left(A^{v} X B^{2-v}+A^{2-v} X B^{v}\right) d v\right\| \\
& \leq \frac{1}{2}\left\|2 A X B+A^{r} X B^{2-r}+A^{2-r} X B^{r}\right\| \leq\left\|A^{r} X B^{2-r}+A^{2-r} X B^{r}\right\| .
\end{aligned}
$$

Further,

$$
\lim _{r \rightarrow 1} \frac{1}{|2-2 r|}\left\|\int_{r}^{2-r}\left(A^{v} X B^{2-v}+A^{2-v} X B^{v}\right) d v\right\|=2\|A X B\| .
$$

From Theorem 3.1 we have the following integral inequalities as an improvement of inequality (1.1).

Corollary 3.3. Let $A, B, X \in M_{n}$ with $A, B$ positive semidefinite and $r \in[0,1]$. Then

$$
\begin{aligned}
2\left\|A^{\frac{1}{2}} X B^{\frac{1}{2}}\right\| & \leq \frac{1}{|1-2 r|}\left\|\int_{r}^{1-r}\left(A^{v} X B^{1-v}+A^{1-v} X B^{v}\right) d v\right\| \| \\
& \leq \frac{1}{2}\left\|A^{\frac{1}{2}} X B^{\frac{1}{2}}+A^{r} X B^{1-r}+A^{1-r} X B^{r}\right\| \| \\
& \leq\left\|\alpha\left(A^{r} X B^{1-r}+A^{1-r} X B^{r}\right)+2(1-\alpha) A^{\frac{1}{2}} X B^{\frac{1}{2}}\right\| \| \text { for } \frac{1}{2} \leq \alpha \leq 1 \\
& \leq\left\|A^{r} X B^{1-r}+A^{1-r} X B^{r}\right\| .
\end{aligned}
$$


Proof. Put $\alpha=r, \beta=1-r$ in inequality (1.3) and Theorem 3.1, respectively, then we get the first inequality and the second inequality. The last inequality follows from the triangle inequality and inequality (1.1). Now we just need to prove the third inequality.

For $0 \leq r \leq 1$, let $\Phi(\alpha)=\left\|\alpha\left(A^{r} X B^{1-r}+A^{1-r} X B^{r}\right)+2(1-\alpha) A^{\frac{1}{2}} X B^{\frac{1}{2}}\right\|$ on $\left[\frac{1}{2}, 1\right]$. Then

$$
\begin{aligned}
\Phi\left(\frac{1}{2}\right) & =\frac{1}{2}\|\| A^{r} X B^{1-r}+A^{1-r} X B^{r}+2 A^{\frac{1}{2}} X B^{\frac{1}{2}}\|\| \\
& =\left\|\left(1-\frac{1}{2 \alpha}\right)\left(2 A^{\frac{1}{2}} X B^{\frac{1}{2}}\right)+\frac{1}{2 \alpha}\left[\alpha\left(A^{r} X B^{1-r}+A^{1-r} X B^{r}\right)+2(1-\alpha) A^{\frac{1}{2}} X B^{\frac{1}{2}}\right]\right\| \| \\
& \leq\left(1-\frac{1}{2 \alpha}\right)\left\|\mid 2 A^{\frac{1}{2}} X B^{\frac{1}{2}}\right\|+\frac{1}{2 \alpha}\left\|\alpha\left(A^{r} X B^{1-r}+A^{1-r} X B^{r}\right)+2(1-\alpha) A^{\frac{1}{2}} X B^{\frac{1}{2}}\right\| \| \\
& \leq\left(1-\frac{1}{2 \alpha}\right) \Phi\left(\frac{1}{2}\right)+\frac{1}{2 \alpha}\left\|\alpha\left(A^{r} X B^{1-r}+A^{1-r} X B^{r}\right)+2(1-\alpha) A^{\frac{1}{2}} X B^{\frac{1}{2}}\right\| .
\end{aligned}
$$

Therefore, for $\frac{1}{2} \leq \alpha \leq 1$, we have the desired inequality as follows.

$$
\frac{1}{2}\left\|A^{r} X B^{1-r}+A^{1-r} X B^{r}+2 A^{\frac{1}{2}} X B^{\frac{1}{2}}\right\| \leq\left\|\alpha\left(A^{r} X B^{1-r}+A^{1-r} X B^{r}\right)+2(1-\alpha) A^{\frac{1}{2}} X B^{\frac{1}{2}}\right\| .
$$

Remark 3.4. The fact that the function $\Phi(\alpha)$ is non-decreasing on $\left[\frac{1}{2}, 1\right]$ can be proved in the same way as in Theorem 2.1.

By the symmetry of the integral function and Theorem 3.1 we have the following corollary:

Corollary 3.5. Let $A, B, X \in M_{n}$ with $A, B$ positive semidefinite and $r \in[0,1]$. Then

$$
\begin{aligned}
& \frac{1}{|1-2 r|}\left\|\int_{r}^{1-r}\left(A^{v} X B^{1-v}+A^{1-v} X B^{v}\right) d v\right\| \| \\
& \quad \leq \frac{1}{4}\left\|4 A^{\frac{1}{2}} X B^{\frac{1}{2}}+A^{1-r} X B^{r}+A^{r} X B^{1-r}+A^{\frac{1+2 r}{4}} X B^{\frac{3-2 r}{4}}+A^{\frac{3-2 r}{4}} X B^{\frac{1+2 r}{4}}\right\| \\
& \leq\left\|A^{1-r} X B^{r}+A^{r} X B^{1-r}\right\| .
\end{aligned}
$$

Proof. Since $\frac{1+2 r}{4}=\frac{1}{2}\left(\frac{1}{2}+r\right)$ and $\left\|A^{1-r} X B^{r}+A^{r} X B^{1-r}\right\|$ is convex on $[0,1]$, we have

$$
\begin{aligned}
& \frac{1}{|1-2 r|}\left\|\int_{r}^{1-r}\left(A^{v} X B^{1-v}+A^{1-v} X B^{v}\right) d v\right\| \| \\
& =\frac{2}{|1-2 r|}\left\|\int_{r}^{\frac{1}{2}}\left(A^{v} X B^{1-v}+A^{1-v} X B^{v}\right) d v\right\| \\
& \leq \frac{1}{4}\left\|2 A^{\frac{1}{2}} X B^{\frac{1}{2}}+A^{1-r} X B^{r}+A^{r} X B^{1-r}+2 A^{\frac{1+2 r}{4}} X B^{\frac{3-2 r}{4}}+2 A^{\frac{3-2 r}{4}} X B^{\frac{1+2 r}{4}}\right\| \\
& \leq \frac{1}{4}\left\|2 A^{\frac{1}{2}} X B^{\frac{1}{2}}+A^{1-r} X B^{r}+A^{r} X B^{1-r}\right\|+\frac{1}{2}\left\|A^{\frac{1+2 r}{4}} X B^{\frac{3-2 r}{4}}+A^{\frac{3-2 r}{4}} X B^{\frac{1+2 r}{4}}\right\| \\
& \leq \frac{1}{4}\left\|2 A^{\frac{1}{2}} X B^{\frac{1}{2}}+A^{1-r} X B^{r}+A^{r} X B^{1-r}\right\|+\frac{1}{4}\left\|A^{\frac{1}{2}} X B^{\frac{1}{2}}\right\| \\
& \quad+\frac{1}{4}\left\|A^{1-r} X B^{r}+A^{r} X B^{1-r}\right\| \\
& \leq\left\|A^{1-r} X B^{r}+A^{r} X B^{1-r}\right\| .
\end{aligned}
$$


Acknowledgement. This research was supported by the Basic Science and Technological Frontier Project of Henan Province(No. 132300410261, No. 142300410167).

\section{REFERENCES}

1. K.M.R. Audenaert, A characterization of anti-Löwner function, Proc. Amer. Math. Soc. 139 (2011), no. 12, 4217-4223.

2. R. Bhatia and C. Davis, More matrix forms of the arithmetic-geometric mean inequality, SIAM J. Matrix Anal. Appl. 14 (1993), 132-136.

3. R.A. Horn and C.R. Johnson, Topics in Matrix Analysis, Cambridge University Press, Cambridge, 1990.

4. R. Kaur, M.S. Moslehian, M. Singh and C. Conde, Further refinements of the Heinz inequality, Linear Algebra Appl. 447 (2014), 26-37.

5. F. Kittaneh, On the convexity of the Heinz means, Integral Equation Operator Theory 68 (2010), no. $4,519-527$.

6. M.K. Kwong, Some results on matrix monotone functions, Linear Algebra Appl. 118 (1989), 129-153.

7. M.K. Kwong, On the definiteness of the solutions of certain matrix equations, Linear Algebra Appl. 108 (1988), 177-197.

8. H. Najafi, Some results on Kwong functions and related inequalities, Linear Algebra Appl. 439 (2013), no. 9, 2634-2641.

9. M. Singh and H.L. Vasudeva, Norm inequalities involving matrix monotone functions, Math. Inequal. Appl. 7 (2004), no.4, 621-627.

10. S.H. Wang, L.M. Zou and Y.Y. Jiang, Some inequalities for unitarily invariant norms of matrices, J. Inequal. Appl. 2011 (2011), no. 10, 1-10.

11. X.Z. Zhan, Inequalities for unitarily invariant norms, SIAM. J. Matrix Anal. Appl. 20 (1998), no. $2,466-470$.

1 College of Mathematics and Information Science, Henan Normal University, XinXiANG, Henan, 453007, China.

E-mail address: zuodke@yahoo.com

2 Department of Mathematics, Osaka Kyoiku University, Asahigaoka, Kashiwara, OsAKA 582-8582, JAPAN.

E-mail address: yukis@cc.osaka-kyoiku.ac.jp

E-mail address: mfujii@cc.osaka-kyoiku.ac.jp 